\title{
What does the Sport Emotion Questionnaire Measure in Terms of Core Affect?
}

\author{
Alexander T. Latinjak ${ }^{1}$, Kristina Cook $^{2}$ and Víctor López-Ros ${ }^{1}$ \\ 1. School of Health and Sport, University of Girona, Salt 17190, Catalonia, Spain \\ 2. Deportment of Physical Education and Sport Sciences, University of Thessaly, Karies 42100, Trikala, Greece \\ 3. Department of Education and Psychology, University of Girona, Girona 17071, Catalonia, Spain
}

Received: October 19, 2013/ Accepted: November 22, 2013 / Published: December 31, 2013.

\begin{abstract}
The purpose of the present study was to use the dimensional approach to understand what an emotion questionnaire based on the basic emotion perspective, such as the SEQ (Sport Emotion Questionnaire), measures in terms of core affect. Therefore, 51 athletes $\left(M_{\text {age }}=19.73\right.$; S.D. $\left.=1.69\right)$ rated the underlying affective dimensions (valence, arousal and time perspective) of the 22 emotion concepts used as items in the SEQ. The analyses of variance and paired samples post-hoc comparisons revealed that each scales' items measure similar core affects and that most scale's underlying core affects are different one from another. Only the excitement and happiness scales were not clearly distinct in terms of core affect. Overall, the results forwarded theoretical validation of the SEQ and exemplified the utility of dimensional models of core affect and emotion concepts as a theoretical framework for the measurement of emotions in research. Based on the study, it is suggested that conciliating the basic emotion and dimensional approach will enhance the knowledge about sport emotions, their antecedents and consequences.
\end{abstract}

Key words: Emotions, measurement, sports, time perspective.

\section{Introduction}

Research in sport science has considered a wide range of emotions [1]. In order to provide researchers with trustworthy tools for their studies, several emotion questionnaires have been developed. On the one hand, specific-emotion measures help to inquire into emotions such as anxiety (Sport Anxiety Scale [2]) or anger (Competitive Aggressiveness and Anger Scale [3]). On the other, non-specific-emotion questionnaires help to inquire into a wider range of emotions. For instance, Jones, Lane, Bray, Uphill and Catlin designed and validated SEQ (Sport Emotion Questionnaire [4]) to assess five emotions of particular relevance to sport settings: anger anxiety dejection excitement and happiness. Showing good evidence of validity and internal consistency, the SEQ has been repeatedly

Corresponding author: Alexander T. Latinjak, Ph.D., associate professor, research field: emotion, self-talk and thoughts. E-mail: alexander.latinjak@cadscrits.udg.edu. applied throughout the past years, studying, for example, (a) the effects of causal attributions on post-competition emotions [5], (b) the effects of emotions on attention, concentration and performance [6], (c) the effects of positive reflection on emotions [7], and (d) the mediating role of perceived performance in the relation between achievement goals and emotions [8]. Thus, the SEQ has possibly helped to advance the knowledge about the antecedents and consequences of emotions in sports.

According to some researchers [9], the SEQ and all aforementioned studies adapt a point of view called "basic emotion theory". In line with basic emotion theory, human nature includes a small number of qualitatively distinct emotions, such as anger, fear or happiness. Even though basic emotion theory represents a plausible approach to emotions that has stimulated the gathering of much evidence, other researchers opted for a different perspective. Instead of considering separate 
emotions, researchers in line with Russell consider several dimensions of core affect. Core affect is defined as "a pre-conceptual primitive process, a neuro-physiological state, accessible to consciousness as a simple non-reflective feeling: feeling good or bad, feeling lethargic or energized". Hence, the two dimensions consistently considered in core affect literature are valence and arousal. One of the main differences between emotions and core affect is that the latter is continuously varying over time, whilst emotions appear and disappear [10]. In a way, a state of core affect that changes, slowly or rapidly, from birth until death, has been reached. Nevertheless, specific states of core affect can be identified by the individual and categorized by specific emotion concepts. For instance, people say that they are excited when they feel good and energized, relaxed when they feel good and lethargic, angry when they feel bad and energized and sad when they feel bad and lethargic [11].

Some researchers have replaced the concept of emotion for the concept of core affect [12]. Similarly, they considered that concepts such as anxious or angry are used to identify specific core affects and to share ones' feelings with others [13, 14]. Taking into account the link between how they feel and the words they use to identify those feelings, the purpose of the study was to explore which affective states the 22 emotion concepts used as items in the SEQ represent. Put another way, the SEQ's items were translated into core affect. Since some authors, including Jones et al. [4], suggested that valance and arousal alone might be insufficient to distinguish some relevant emotions such as anger and anxiety [15], a third dimension namely time perspective (that ranges between anticipatory and retrospective) which has shown to add variability to the original model [13, 16, 17] was used. Consequently, what degree each of the 22 items represented positive or negative, activated or deactivated and anticipatory or retrospective core affects was explored.

The study stated the following hypotheses: Regarding the internal structure of the SEQ in terms of core affect, the emotion concepts within each scale were expected to be similar in terms of valence, arousal and time perspective; and the emotion concepts of the anxious, dejected and angry scales to be more negative in valence compared to the ones of the excited and happy scales [4]. Moreover, the emotion concepts of the anger and anxiety scale were expected to be similar in terms of valence and arousal, but dissimilar in terms of perspective [13]. Specifically, based on Latinjak et al.'s preliminary findings, the anger scale's emotion concepts were expected to represent more retrospective core affects and the anxiety scale's ones more anticipatory core affects.

\section{Materials and Methods}

\subsection{Participants}

Fifteen female and 36 male American athletes ( $M_{\text {age }}$ = 19.73; S.D. $=1.69)$ who were playing soccer $(n=26)$, basketball $(n=14)$ and tennis $(n=11)$ and competing on a regional $(n=10)$, state $(n=14)$, national $(n=13)$ and international $(n=14)$ level have participated in the study. They were approached in their regular training facilities and they all gave their informed consent to participate in the study. Ethical approval for the study was granted by the local ethic comity.

\subsection{Procedure and Measures}

Once the participants agreed to participate, they received a booklet that contained 22 groups of three questions. Each group of questions was preceded by one emotion concept used in the SEQ and an introducing sentence (e.g., Excited. Usually, in a situation they feel excited ...). The SEQ uses (a) nervous, anxious, tense, apprehensive ,and uneasy to measure anxiety; (b) unhappy, sad, upset, dejected and disappointed to measure dejection; (c) annoyed, irritated, furious and angry to measure anger; (d) enthusiastic, excited, energetic, and exhilarated to measure excitement; and (e) joyful, pleased, cheerful, and happy to measure happiness. The three questions following the stem were the same for each emotion 
concept: Firstly, the participants rated valence from 1 (very unpleasant) to 7 (very pleasant). Secondly, they rated arousal from 1 (very deactivated) to 7 (very activated). Lastly, they rated time perspective from 1 (retrospective) to 7 (anticipatory).

\section{Results}

In order to represent the five basic emotions measured by the SEQ inside the three dimensional models of core affect (Fig. 1), it was calculated their descriptive statistics for valence, arousal and time perspective (Table 1). In order to represent the area of each SEQ scale in the dimensional space, it was used their emotion concepts' upper and lower bounds of the 95\% confidence interval in each dependent measure. Hence, for each scales' area (a) the lowest lower bound value and the highest upper bound value in valence defined its height, (b) the lowest lower bound value and the highest upper bound in activation defined its depth and (c) the lowest lower bound value and the highest upper bound value in time perspective defined its width.

\subsection{Intra-scale Comparisons}

Firstly, the emotion concepts of each scale seem to be relatively close on by the other in the tri-dimensional model. According to dependent samples $t$ tests using the Bonferroni correction, (a) only four significant differences out of 30 comparisons (10 paired comparisons $\times 3$ dependent variables) indicated that the anxiety scale contained similar emotion concepts, (b) only two significant differences out of 30 . comparisons indicated that the dejection scale contained similar emotion concepts, (c) only two significant differences out of 18 comparisons indicated that the anger scale contained similar emotion concepts,

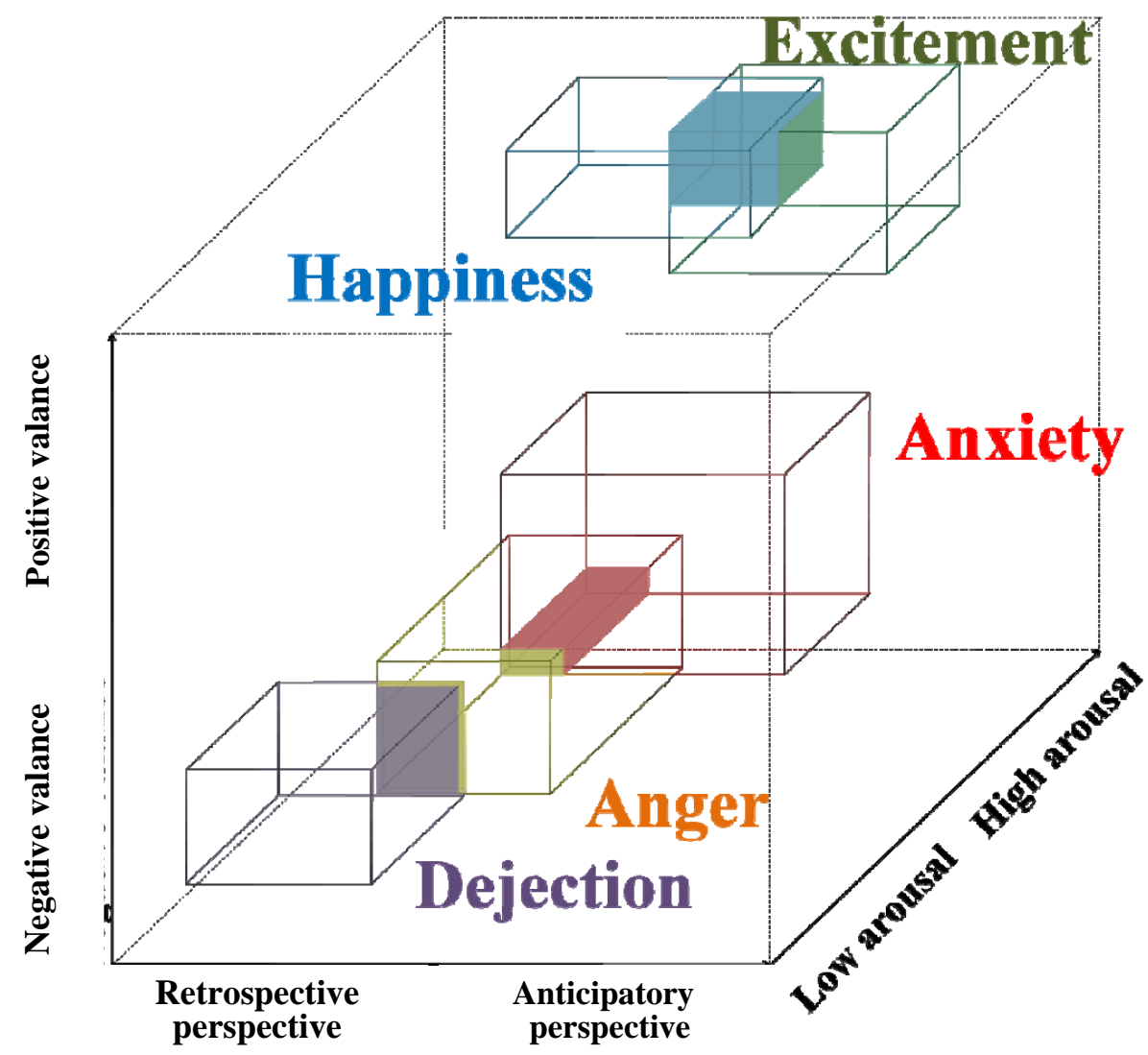

Fig. 1 Placement of the five sport-relevant emotions measured in the sport emotion questionnaire in the tri-dimensional model of core affect. Areas represent the five scales and are defined by the scales emotion concepts' highest upper bound and lowest lower bound values of the $95 \%$ confidence interval. 
Table 1 Mean scores, standard deviations and confidence interval for valence, arousal and time perspective of all 22 emotion concepts of the SEQ.

\begin{tabular}{|c|c|c|c|c|c|c|c|c|c|c|c|c|}
\hline \multirow[b]{3}{*}{ Emotion concepts } & \multicolumn{4}{|c|}{ Vanlence } & \multirow{3}{*}{$M(1-7)$} & \multirow{3}{*}{$S D$} & \multicolumn{2}{|c|}{ Activation } & \multicolumn{2}{|c|}{ Tim } & \multicolumn{2}{|c|}{ Perspective } \\
\hline & \multirow{2}{*}{$M(1-7)$} & \multirow{2}{*}{$S D$} & \multicolumn{2}{|c|}{$95 \% \mathrm{CI}$} & & & \multicolumn{2}{|c|}{$95 \% \mathrm{CI}$} & \multirow{2}{*}{$M(1-7)$} & \multirow{2}{*}{$S D$} & \multicolumn{2}{|c|}{$95 \% \mathrm{CI}$} \\
\hline & & & LB & UB & & & LB & UB & & & LB & UB \\
\hline \multicolumn{13}{|l|}{ Anxiety scale } \\
\hline Anxious & 3.86 & 1.04 & 3.57 & 4.16 & 4.67 & 1.41 & 4.27 & 5.06 & 5.33 & 1.65 & 4.87 & 5.80 \\
\hline Uneasy & 2.78 & 1.14 & 2.47 & 3.10 & 3.92 & 1.41 & 3.52 & 4.32 & 3.80 & 1.84 & 3.29 & 4.32 \\
\hline Nervous & 3.18 & 0.91 & 2.92 & 3.43 & 4.55 & 1.32 & 4.18 & 4.92 & 5.45 & 1.47 & 5.04 & 5.87 \\
\hline Tense & 2.94 & 1.10 & 2.63 & 3.25 & 4.47 & 1.21 & 4.13 & 4.81 & 4.33 & 1.60 & 3.88 & 4.78 \\
\hline Apprehensive & 4.04 & 1.30 & 3.68 & 4.40 & 4.16 & 1.30 & 3.79 & 4.52 & 4.57 & 1.15 & 4.24 & 4.89 \\
\hline \multicolumn{13}{|l|}{ Dejection scale } \\
\hline Disappointed & 1.61 & 0.75 & 1.40 & 1.82 & 2.39 & 1.25 & 2.04 & 2.74 & 1.59 & 0.94 & 1.32 & 1.85 \\
\hline Unhappy & 1.75 & 0.91 & 1.49 & 2.00 & 2.57 & 1.32 & 2.20 & 2.94 & 2.41 & 1.37 & 2.03 & 2.80 \\
\hline Sad & 1.86 & 0.83 & 1.63 & 2.10 & 2.02 & 1.10 & 1.71 & 2.33 & 2.16 & 1.21 & 1.82 & 2.50 \\
\hline Upset & 2.02 & 0.93 & 1.76 & 2.28 & 3.02 & 1.36 & 2.64 & 3.40 & 2.61 & 1.33 & 2.23 & 2.98 \\
\hline Dejected & 2.22 & 0.97 & 1.94 & 2.49 & 2.59 & 1.28 & 2.23 & 2.95 & 2.63 & 1.37 & 2.24 & 3.01 \\
\hline \multicolumn{13}{|l|}{ Angry scale } \\
\hline Furious & 1.82 & 1.26 & 1.47 & 2.18 & 5.12 & 2.04 & 4.55 & 5.69 & 2.78 & 1.84 & 2.27 & 3.30 \\
\hline Annoyed & 2.41 & 1.13 & 2.09 & 2.73 & 3.92 & 1.47 & 3.51 & 4.33 & 3.63 & 0.77 & 3.41 & 3.85 \\
\hline Angry & 2.04 & 1.22 & 1.70 & 2.38 & 4.84 & 1.63 & 4.39 & 5.30 & 2.92 & 1.40 & 2.53 & 3.32 \\
\hline Irritated & 2.06 & 0.90 & 1.81 & 2.31 & 3.75 & 1.61 & 3.29 & 4.20 & 3.04 & 1.13 & 2.72 & 3.36 \\
\hline \multicolumn{13}{|l|}{ Excitement scale } \\
\hline Excited & 5.80 & 1.28 & 5.44 & 6.16 & 6.24 & 1.01 & 5.95 & 6.52 & 5.29 & 1.70 & 4.82 & 5.77 \\
\hline Energetic & 6.37 & 0.82 & 6.14 & 6.60 & 6.73 & 0.57 & 6.57 & 6.89 & 4.43 & 1.47 & 4.02 & 4.85 \\
\hline Enthusiastic & 6.35 & 0.91 & 6.10 & 6.61 & 6.24 & 1.03 & 5.95 & 6.53 & 5.37 & 1.31 & 5.00 & 5.74 \\
\hline Exhilarated & 5.61 & 1.22 & 5.27 & 5.95 & 5.90 & 1.10 & 5.59 & 6.21 & 4.20 & 1.43 & 3.79 & 4.60 \\
\hline \multicolumn{13}{|l|}{ Happiness scale } \\
\hline Cheerful & 6.31 & 0.948 & 6.05 & 6.58 & 6.10 & 1.06 & 5.80 & 6.40 & 4.37 & 1.25 & 4.02 & 4.72 \\
\hline Нарру & 6.49 & 0.758 & 6.28 & 6.70 & 6.08 & 0.85 & 5.84 & 6.32 & 4.29 & 1.74 & 3.81 & 4.78 \\
\hline Joyful & 6.37 & 0.720 & 6.17 & 6.58 & 5.88 & 0.93 & 5.62 & 6.14 & 4.24 & 1.61 & 3.78 & 4.69 \\
\hline Pleased & 6.18 & 0.953 & 5.91 & 6.45 & 5.41 & 1.22 & 5.07 & 5.76 & 3.02 & 1.63 & 2.56 & 3.48 \\
\hline
\end{tabular}

Valence ranged from negative to positive; arousal from deactivated to activated; and perspective from past related to future related. CI $=$ Confidence interval; $\mathrm{LB}=$ Lower bound; UB = Upper bound. Bold values represent the lower or upper bound values used in Fig. 1 to create each scale's area inside the dimensional model. Consequently, one lower and one upper bound value were used for each dependent variable in each scale.

(d) no significant differences out of 18 comparisons indicated that the excitement scale contained similar emotion concepts and (e) no significant differences out of 18 comparisons indicated that also the happiness scale contained similar emotion concepts. However, some emotion concepts have shown to be distinct to others used in the same scale in more than one dependent variable. Uneasy $(A \times 2)$ was rated more negative than anxious $(\mathrm{A} \times 1 ; p=0.002)$ and apprehensive ( $\mathrm{A} \times 5 ; p=0.045)$; and more past related than nervous $(\mathrm{A} \times 3 ; p=0.038)$. Moreover, upset (D4) was rated more activated than sad (D3; $p=0.002)$ and more future related than disappointed (D1; $p=0.003)$.

\subsection{Inter-scale Comparisons}

Secondly, each scale seems to represent a relatively reduced and localized area inside the tri-dimensional model. Taking the seven points of the Likert scale as basic units, the area of all five emotion concepts inside the model (total area 343 units $^{3}$ ) was calculated: Dejection (3.14 units ${ }^{3}$ ) was identified as a negative, deactivated and retrospective core affect, Anger (4.52 units $^{3}$ ) as a negative, activated and retrospective one, Anxiety (7.66 units ${ }^{3}$ ) as a negative, activated and 
anticipatory one and both Excitement (3.45 units ${ }^{3}$ ) and Happiness (2.33 units ${ }^{3}$ ) as positive, activated and anticipatory ones.

Valence. Further dependent samples $t$ tests revealed that all emotion concepts of all negative scales were rated as significantly more negative as the emotion concepts of the positive scales (112 significant differences; all $p$ 's $<0.010$ ). Moreover, the emotion concepts of the Anxiety scale were rated as more positive compared to the emotion concepts of the Anger (13 significant differences out of 20) and Dejection scale (20 significant differences out of 25). The Anger and Dejection scales' emotion concepts were rated as similar in terms of Valence (only one significant difference out of 20).

Arousal. In regard to activation, the emotion concepts of the Dejection scale were rated as less activated compared to all other scales: Anger (14 significant differences out of 20), Anxiety (21 significant differences out of 25), Happiness (all 20 differences were significant; all $p$ 's $<0.001$ ) and Excitement (all 20 differences were significant; all $p$ 's $<0.001$ ). Moreover, the emotion concepts of the Happiness and the Excitement scale were rated as more activated as the ones of the Anger (Happiness: eight significant differences out of 16; Excitement: 12 significant differences out of 16) and Anxiety (Happiness: 16 significant differences out of 20; Excitement: all 20 differences were significant, all $p$ 's $<0.010)$ scale. Lastly, the emotion concepts were similar in terms of Valence between the Anger and Anxiety scale (no significant differences) and between the Happiness and Excitement scale (only four significant differences out of 20).

Time Perspective. In regard to Time Perspective, the emotion concepts of the Anxiety, Happiness and Excitement scale were rated as similar in terms of Time Perspective (only three significant differences out of 56). Moreover, the emotion concepts of the Anger scale were rated as more retrospective than the ones of the Anxiety (13 significant differences out of 20),
Happiness (11 significant differences out of 16) and Excitement scale (seven significant differences out of 16). Similarly, the emotion concepts of the Dejection scale were also rated as more past retrospective than the ones of the Anxiety (all 25 differences were significant, all $p$ 's $<0.010$ ), Happiness (16 significant differences out of 20) and Excitement scale (all 20 differences were significant, all $p$ 's $<0.010$ ). Lastly, some of the emotion concepts of the Anger scale were rated as more anticipatory as the ones of the Dejection scale (seven significant differences out of 20).

\subsection{Intersections between Scales}

Thirdly, there are three intersections between different scales' areas. The smallest intersection was found between the Dejection and the Anger scale (0.08 units $\left.^{3}\right)$. The area of the intersection represents the $2.55 \%$ and $1.77 \%$ of Dejection and Anger's total areas, respectively. A larger intersection was observed between the Anger and the Anxiety scale (0.22 units $\left.{ }^{3}\right)$. The area of the intersection represents the $4.87 \%$ and 2.87\% of Anger and Anxiety's areas, respectively. Since the Anxiety scale was rated as more positive and more future related than the Anger scale, the emotion concept uneasy $(\mathrm{A} \times 2)$ could be responsible for the interaction. Uneasy $(A \times 2)$ was rated as more negative and more retrospective than the other four emotion concepts in the Anxiety scale. Lastly, the biggest intersection was found between the Excitement and the Happiness scale $\left(0.56\right.$ units $\left.^{3}\right)$. The area of the intersection represents the $16.23 \%$ and $24.03 \%$ of Excitement and Happiness' areas, respectively. Based on the results of the post-hoc comparisons where no differences were identified between both scales in terms of its underlying dimensions, it seems that they measure similar core affects.

\section{Conclusions}

The purpose of the present study was to use the dimensional approach to understand what a basic-emotion measure, such as the SEQ, measures in 
terms of core affect. Overall, the results support the hypothesis: the emotion concepts within each scale represented similar core affects in terms of valence, arousal and perspective; and the emotion concepts of the anxious, dejected and angry scales represented more negative core affects in terms of valence compared to the ones of the excited and happy scales. Moreover, the emotion concepts of the anger and anxiety scale represented dissimilar core affects in terms of perspective being the anger scale's emotion concepts more retrospective than the anxiety scale's ones.

Consequently, there is a strong support for the assumption that the SEQ measures both positive and negative emotions, as intended by Ref. [4]. However, both positive scales were not distinct in terms of their underlying core affect. Contradictorily, in two studies employing the SEQ, Allen et al. evidenced that happiness increases greater after success in comparison to excitement [5], suggesting that past success influences happiness to a greater degree. In the study, visual inspection reveals that Happiness seemed to be in deed more retrospective compared to excitement, but statistical support was not obtained. The incongruence outlines the need for more research in the area. On the other hand, there are parts of the tri-dimensional model that were not covered by the instrument. According to the findings, the SEQ fails to measure four typical core affects, which would be identified by emotion concepts such as confidence (positive, low and anticipatory), relief (positive, low and retrospective), euphoria (positive, high and retrospective) and hopelessness (negative, low and anticipatory).

Once the SEQ's emotion concepts were placed in terms of valence, arousal and time perspective, it would be capable of understanding former results under a different point of view. For instance, Allen et al. [7] have studied the effects of positive reflection on attributions, emotions and self-efficacy. Their results indicated that reflecting back on positive elements of performance was useful for developing desirable attributions, but not necessarily for promoting self-efficacy or positive emotions. Now it can be rephrased that positive reflection did not influence positive-high-future emotions. However, common sense suggests that positive reflection might be a useful tool to influence past related positive core affects, represented by emotion concepts such as euphoria, satisfaction or relief.

Generally, the study of the underlying core affect dimensions of the emotion concepts used in the SEQ [4] forwarded theoretical validation of the instrument. Moreover, the study has also supported the dimensional approach and, specifically, exemplified the utility of the tri-dimensional model of core affect and emotion concepts $[13,17]$ as a theoretical framework for the measurement of emotions in research. It has allowed to approach an instrument based on basic emotion theory from a dimensional perspective. The gathered evidence should not undermine previous findings from a basic point of view, but enhance their understanding. Future studies should further link measures and studies of specific emotions to core affect, in order to (a) gather evidence in regard to the validity of the dimensional approach as a theoretical framework (b) improve the design of both emotion instruments and research designs for studies involving emotion and (c) enhance the knowledge about sport emotions, their antecedents and consequences.

\section{Acknowledgments}

The research was supported in part by (a grant from) the Spanish Ministry of Science and Innovation (Ministerio de Ciencia e Innovación) (DEP2010-15561), the Private Foundation EUSES, and the "Càtedra d'Esport i Educació Física de la Universitat de Girona”.

\section{References}

[1] C. Sève, L. Ria, G. Poizat, J. Saury, M. Durand, Performance-induced emotions experienced during high-stakes table tennis matches, Psychology of Sport and 
Exercise 8 (2007) 25-46.

[2] R.E. Smith, F.L. Smoll, R.W. Schultz, Measurement and correlates of sport specific cognitive and somatic trait anxiety: the sport anxiety scale, Anxiety Research 2 (1990) 263-280.

[3] J.P. Maxwell, E. Moores, The development of a short scale for measuring aggressiveness and anger in competitive athletes, Psychology of Sport and Exercise 8 (2007) 179-193.

[4] M.V. Jones, A.M. Lane, S.R. Bray, M. Uphill, J. Catlin, Development and validation of the sport emotion questionnaire, Journal of Sport and Exercise Psychology 27 (2005) 407-431.

[5] M.S. Allen, M.V. Jones, D. Sheffield, Causal attribution and emotion in the days following competition, Journal of Sports Sciences 27 (2009) 461-468.

[6] R.L. Vast, R.L. Young, P.R. Thomas, Emotions in sport: Perceived effects on attention, concentration, and performance, Australian Psychologist 45 (2010) 132-140.

[7] M.S. Allen, M.V. Jones, D. Sheffield, The influence of positive reflection on attributions, emotions, and self-efficacy, The Sport Psychologist 24 (2010) 211-226.

[8] A.J. Dewar, M. Kavussanu, Achievement goals and emotions in golf: the mediating and moderating role of perceived performance, Psychology of Sport and Exercise 12 (2011) 525-532.

[9] J.A. Russell, Emotion, core affect, and psychological construction, Cognition and Emotion 23 (2009) 1259-1283.

[10] E. Diener, A. Iran-Nejad, The relationship in experience between various types of affect, Journal of Personality and Social Psychology 50 (1986) 1031-1038.

[11] J.A. Russell, L.F. Barrett, Core affect, prototypical emotional episodes, and other things called emotion: Dissecting the elephant, Journal of Personality and Social Psychology 76 (1999) 805-819.

[12] J.A. Russell, From a psychological constructionist perspective, in: P. Zachar, R. Ellis (Eds.), Categorical Versus Dimensional Models of Affect: A Seminar on the Theories of Panksepp and Russell, John Benjamins Publishing, Amsterdam, 2012, pp. 79-118.

[13] A.T. Latinjak, V. López-Ros, R. Font-Lladó, A descriptive study of emotion concepts using dimensional models of core affect, Faculty of Psychology (in press).

[14] P.M. Niedenthal, Emotion concepts, in: M. Lewis, J.M. Haviland-Jones, L.F. Barrett (Eds.), Handbook of Emotions, 3rd ed., The Guilford Press, New York, 2008, pp. 587-600.

[15] J.R.J. Fontaine, K.R. Scherer, E.B. Roesch, P.C. Ellsworth, The world of emotion is not two-dimensional, Psychological Science 18 (2007) 1050-1057.

[16] P. Ballart, N. Broo, B. Juan, A. Valls, A.T. Latinjak, Situational motivation and affective state in physical activity classes, Motricidad, European Journal of Human Movement 29 (2012) 147-158.

[17] A.T. Latinjak, The underlying structure of emotions: a tri-dimensional model of core affect and emotion concepts for sports, Revista Iberoamericana de Psicología del Ejercicio y el Deporte 7 (2012) 71-87. 